\title{
Autonome Fahrzeuge und autonomes Fahren im Bereich des Gütertransportes
}

\author{
Heike Flämig
}

\section{Inhaltsverzeichnis}

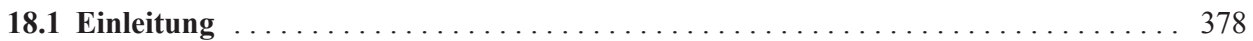

18.2 Entwicklungsgeschichte fahrerloser und autonomer Transportsysteme . . . . . . 379

18.2.1 Fahrerlose Transportsysteme im Innenbereich . . . . . . . . . . . . . . . . . . 379

18.2.2 Fahrerlose Transportfahrzeuge im Außenbereich . . . . . . . . . . . . 381

18.2.3 Autonome Fahrzeuge für den Straßengütertransport außerhalb

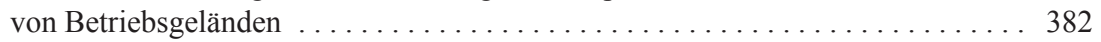

18.2.4 Entwicklungen autonomen Fahrens und autonomer Fahrzeuge

in den anderen Verkehrsträgern . . . . . . . . . . . . . . . . . . . . . . . . . . 382

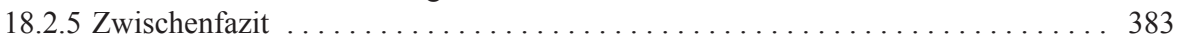

18.3 Anwendungsfälle im Bereich des autonomen Gütertransports . . . . . . . . . . . . 384

18.3.1 Exkurs: Automatisierungsgrade des autonomen Gütertransports . . . . . . . . . 385

18.3.2 Anwendungsfälle des autonomen Gütertransports $\ldots \ldots \ldots \ldots \ldots \ldots \ldots . \ldots \ldots 5$

18.3.3 Autobahnpilot mit Fahrer und freier Navigation $\ldots \ldots \ldots \ldots \ldots \ldots \ldots . \ldots \ldots 6$

18.3.4 Vehicle-on-Demand als Autobahnfahrt ohne Fahrer mit freier Navigation . . . . . 387

18.3.5 Vollautomat mit Verfügbarkeitsfahrer - Follow-Me-Fahrzeug . . . . . . . . . . . . . . . 389

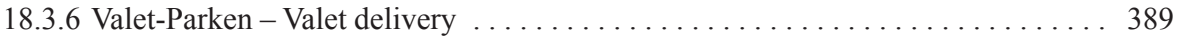

18.4 Veränderungen in der Supply Chain durch einen höheren Automatisierungsgrad

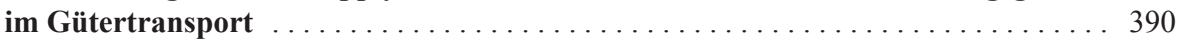

18.5 Erste einzelwirtschaftliche Einschätzungen

von automatisierten Systemen in der Gütertransportkette

H. Flämig ( $ه)$

Technische Universität Hamburg-Harburg, Institut für Verkehrsplanung und Logistik, Deutschland flaemig@tu-harburg.de 


\subsection{Erste gesamtwirtschaftliche Einschätzungen \\ von automatisierten Systemen in der Gütertransportkette . . . . . . . . . . . 393}

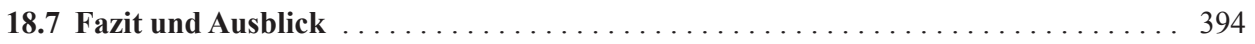

Literatur . . . . . . . . . . . . . . . . . . . . . . . . . . . . 397

\subsection{Einleitung}

Zur Erhöhung von Produktivität, Zuverlässigkeit und Flexibilität der Raumüberwindung nimmt der Automatisierungsgrad der Fahrzeuge sowohl im Bereich der Verkehrsträger als auch im Bereich des innerbetrieblichen Transports kontinuierlich zu. Durch die anwachsende Informationsdichte und Komplexität räumlicher Arbeitsteilung gewinnt die Idee der sich selbststeuernden, dezentralen Einheiten an Bedeutung. Die Konzentration der öffentlichen und wissenschaftlichen Diskussion zum autonomen Fahren mit individuellen Personenkraftwagen vernachlässigt bisher allerdings rund ein Drittel des Verkehrs auf öffentlichen Straßen, nämlich den Wirtschaftsverkehr. Wiederum ein Drittel des Wirtschaftsverkehrs entsteht durch den Transport von Gütern, der notwendig ist, da der Ort der Gütererstellung selten dem Ort der Güternachfrage entspricht. Das Fahren selbst stiftet keinen Mehrwert und ist nur Mittel zum Zweck der Raumüberwindung. Aus diesem Grund wurden bereits in den 1950er-Jahren erste Anwendungen in der innerbetrieblichen Logistik entwickelt, bei denen der Transport ohne Fahrer realisiert werden konnte. Die Entwicklung sogenannter Fahrroboter erfolgte vor allem für spezielle Missionen in gefährlichen oder kaum zugänglichen Gebieten. Automatisierte, fahrerlose und teilweise autonome Fahrzeuge sind also schon seit Längerem für den Transport von Gütern in der Produktion oder in logistischen Systemen im Einsatz.

Ganz grundsätzlich stellt sich die Frage, inwieweit auch im Straßengüterverkehr auf öffentlicher Infrastruktur vollautomatisches Fahren eine sinnvolle Anwendung sein kann. Daran knüpfen sich weitere Fragen an die notwendigen technischen und organisatorischen sowie rechtlichen und sicherheitsbezogenen Voraussetzungen für eine erfolgreiche Umsetzung und sich daraus möglicherweise ergebenden Veränderungen in der Logistik bzw. in Supply Chains und im Güterverkehrssystem.

Das Kapitel nähert sich diesen Fragestellungen zunächst historisch aus der Perspektive der innerbetrieblichen Logistik, da in diesem Zuge die Beweggründe von Unternehmen für die Implementierung von fahrerlosen Transportsystemen gut nachvollziehbar werden und der Erfahrungshintergrund der Unternehmensentscheider offengelegt wird. Anhand von Fallbeispielen aus dem Bereich von Logistik und Güterverkehr werden derzeitige Einsatzfelder aufgezeigt und, soweit bekannt, das für die autonome Fahrt wichtige Navigations- und Sicherheitskonzept sowie die Steuerung beschrieben. Zudem werden aufbauend auf den in Kap. 2 beschriebenen Anwendungsfällen spezifische Anwendungsfälle für den Güterverkehr skizziert. Die sich parallel entwickelnden autonomen Systeme der anderen Verkehrsträger, 
beispielsweise Drohnen, zeigen, dass vollkommen neue Geschäftsmodelle im Bereich der Logistik durch autonome Systeme im Entstehen sind. Die zu erwartenden Veränderungen in den logistischen Prozessen sowie die veränderte Rolle der menschlichen Arbeit in den Systemen werden anhand einer generischen Supply Chain diskutiert. Das Kapitel schließt mit Handlungsempfehlungen und der Benennung des weiteren Forschungsbedarfs.

\subsection{Entwicklungsgeschichte fahrerloser und autonomer Transportsysteme}

Fahrerlose Transportsysteme (FTS) und Fahrerlose Transportfahrzeuge (FTF) kamen erstmals Anfang der 1950er-Jahre in Amerika und rund zehn Jahre später in Deutschland zum Einsatz [1]. Im Vordergrund stand die Optimierung des Materialflusses und die Reduzierung des Personaleinsatzes als logische Konsequenz der sich seit dem frühen 19. Jahrhundert ausbreitenden Ideen zur Erhöhung der Produktivität durch die Verbesserung der Arbeitsabläufe (Taylor), über die Vertaktung der Produktionsstufen (insbesondere geprägt durch das „System vorbestimmter Zeiten“ nach Gilbreth) bis hin zur kontinuierlichen Fließbandfertigung (Ford). Getrieben wurde diese Entwicklung durch die Mechanisierung der Produktion aufgrund einer zunehmenden Technikzentrierung des Managements bis hin zur Vorstellung einer vollständigen Automatisierung (vgl. die historische Nachzeichnung bei [1]). Beginnend mit der Automatisierung der Transporte zwischen Bearbeitungsstufen erfolgte die erste Entwicklung von FTS für den Einsatz in der Produktion und im Lager. Im Vergleich zu automatisierten Fördersystemen, beispielsweise Bandanlagen, sind die Investitionen in der Regel wesentlich geringer und die Flexibilität gegenüber Änderungen im Materialfluss wesentlich höher [2]. Zudem gelten Transporte als unproduktiv, müssen aber in Produktions- und Lagersystemen eine hohe Zuverlässigkeit aufweisen.

\subsubsection{Fahrerlose Transportsysteme im Innenbereich}

Typische Einsatzbereiche von FTS im Innenbereich bestehen einerseits zwischen den Produktions- und Montagestufen und andererseits im Wareneingang und Warenausgang sowie in der Kommissionierzone und im Lagerbereich. Dabei sind FTS und Arbeitsroboter, beispielsweise zum Kommissionieren, Pallettieren etc., häufig baulich miteinander verbunden. In der innerbetrieblichen Logistik war der Begegnungsfall zwischen Mensch und FTS/FTF daher immer vorgesehen, da FTS/FTF in der Regel weitere Funktionen, z. B. als Kommissionierhilfsmittel, übernehmen. Die frühen Personenschutzkonzepte arbeiteten mit taktilen, mechanischen Bügeln bzw. Bumpern [2]. Heute kommen vermehrt Laserscanner zum Einsatz, die in Abhängigkeit von der Fahrgeschwindigkeit bis zu sieben Meter abdecken [2] und häufig mit weiteren Sensortechniken kombiniert werden. Die Stabilisierungsebene spielt im innerbetrieblichen Transport eine untergeordnete Rolle, da das Unternehmen den Untergrund selbst im fahrfähigen Zustand hält. 
Die Bahnführungsebene im Indoor-Bereich der innerbetrieblichen Transporte erfolgte bei den frühen Systemen in der Regel mithilfe von stromführenden Leitern, die im Hallenboden eingelassen waren. Heute werden folgende Typen zur Positionserkennung und Positionierung unterschieden [3]: Auf der einen Seite kommt weiterhin die physische Leitlinie zum Einsatz, ausgeführt als aktiv-induktive Leitspur, als Magnetband oder als optische Leitspur. Auf der anderen Seite wird mit Technologien zur freien Navigation gearbeitet, bei denen die Orientierung mithilfe von Bodenmarken (Metall, Magnet, Transponder) oder auf Basis der Lasertechnologie, bei der die Positionsbestimmung ähnlich funktioniert wie in der Seeschifffahrt (Kreuzpeilung), erfolgt. Neuere Technologien kombinieren Laserscanner und Kamerasysteme mit digitalen Umgebungskarten und machen eine Navigation mittels Umgebungsmerkmalen möglich.

Für die Datenübertragung zwischen den stationären und den mobilen Einheiten eines FTS kam früher die induktive bzw. die Infrarot-Datenübertragung zum Einsatz. Heute sind Schmalbandfunk und immer mehr Breitbandfunk (WLAN) vorherrschend. Die Funkpeilung ermöglicht eine Ortung mittels Indoor-GPS (Global Positioning System) mit einer Genauigkeit bis zu 0,5 Metern und mittels Outdoor-GPS mit einer Genauigkeit von zehn Metern, dGPS (Differential GPS) mit einer Genauigkeit bis zu einem Meter oder dGPS mit Phasenauswertung mit einer Genauigkeit bis zu 0,1 Metern.

Fahrzeuge bekommen ihre Fahraufgaben zugewiesen und werden koordiniert [2]. Das Steuerungskonzept besteht aus einer Leitsteuerung, durch die die Transportauftragsabwicklung mit der Transportauftragsverwaltung, der Fahrzeugdisposition und der Fahrauftragsabwicklung zentral erfolgt. Die Verkehrsleitsteuerung ist ein Teil der Fahrauftragsabwicklung. Die Freigabe einzelner Streckenabschnitte geschieht ähnlich wie im Bahnverkehr in Blockstrecken, die jeweils nur von einem Fahrzeug belegt werden dürfen.

Eine beispielhafte Anlage wurde in einem Distributionszentrum eines Logistikdienstleisters im Jahr 2011 realisiert [3]. Bei der Kommissionierung kommen FTS zum Einsatz, die auch die Kommissionierer mit sich führen. Die Sicherheit des Mitfahrers wird durch verschiedene Maßnahmen hergestellt, die wie beim Bahntransport vom Zugführungspersonal verlangen, dass er eine bestimmte Position mit den Händen und Füßen einnimmt. Andere Betätigungen sind daher während der Fahrt nicht möglich. Das Personenschutzkonzept erfolgt mittels Laserscanner. Für die Navigation wird die Magnetpunktfolge genutzt, und für die Leitsteuerung kommt zur Datenübertragung die WLAN-Technologie zum Einsatz.

Die FTS/FTF-Leitsteuerung erfolgt auch in den neueren Anlagen zentral. Die Erweiterung der Systeme ist daher immer mit einem großen Aufwand verbunden, sodass auch hier zu autonomen, dezentralen Steuerungslösungen geforscht wird.

Ein aktuelles Beispiel ist der autonome Schubmaststapler als Ergebnis des Projekts „marion (mobile, autonome, kooperative Roboter in komplexen Wertschöpfungsketten)“, das im Rahmen des Technologieprogramms „Autonomik“ (Autonome und simulationsbasierte Systeme für den Mittelstand) entwickelt wurde. Die Fahrzeuge bekommen ihre Aufgaben von einem übergeordneten System zugewiesen. Der Stapler führt diese Aufgabe autonom aus und berechnet und entscheidet selbstständig über den optimalen Fahrweg. Er ist mit einem 3-D-Laser, Laserscanner und Kameras zur 3-D-Umgebungserfassung ausge- 
stattet. Durch Sensorenbündel kann der Stapler die Umgebung exakt erfassen, die Objektabmessung und die räumliche Position des Objekts bestimmen.

Eine dezentrale Steuerung stellen beispielsweise [4] vor, die im Rahmen des Projekts „Dezentrale, agentenbasierte Selbststeuerung von Fahrerlosen Transportsystemen (FTS)“ erarbeitet wurde. Darin sind für jedes Fahrzeug verschiedene Agenten modelliert. Die Routenplanung und Auftragsvergabe erfolgt kooperativ. Simulationen ergaben eine Reduzierung der Gesamtstrecke aller FTF und des Leerfahrtenanteils um rund acht Prozent, der Durchlaufzeit um 22 Prozent sowie eine geringe Auslastungserhöhung der FTF [5]. Am Fraunhofer Institut für Materialflusstechnik und Logistik (IML) wurde im Projekt „Schwarmintelligenz für die Logistik“ ein zellulares Transportsystem entwickelt, das auf die Schwarmintelligenz aufbaut. In diesem System erhält der Schwarm (die Transportmittel) die Transportaufträge, das nächstgelegene Transportmittel übernimmt den Auftrag und sucht sich dynamisch den kürzesten Weg.

\subsubsection{Fahrerlose Transportfahrzeuge im Außenbereich}

Typische Einsatzbereiche autonomer Fahrzeuge im Außenbereich, aber auf privatem Gelände, sind beispielsweise FTS für Schwertransporte oder werksinterne Shuttle-Verkehre.

Auf einem Container-Terminal transportieren sogenannte Automated Guided Vehicle (AGV) Container zwischen den Containerbrücken und dem Containerlager [6]. Dadurch sollen Fahrstrecken verkürzt und Leerfahrten reduziert sowie alle Ressourcen optimal ausgelastet werden. Für die Positionsbestimmung werden im Boden eingelassene Transponder genutzt. Die Routenplanung erfolgt selbstständig, ebenso wie der Batteriewechsel. Die Leitsteuerung erfolgt per Datenfunk.

In Deutschland ist ein starker Treiber des führerlosen Lkw ein Unternehmen, das ursprünglich aus der Funktechnik kommt. Im Jahr 2012 wurde beispielsweise durch dieses Unternehmen ein fahrerloser Lkw-Shuttle zwischen dem Produktions- und Logistikgebäude auf dem Werksgelände einer Molkerei realisiert [7]. Die Be- und Endladung der mit Verpackungsmaterial bzw. Frischprodukten beladenen Euro- und Industriepaletten erfolgt automatisch. Zur Spurführung dienen Transponder in der Fahrbahn. Unter der Zugmaschine ist ein Sensor angebracht, der die Markierung im Boden zur Positionierung und Wegfindung nutzt. Unterstützt durch lenkbare Hinterachsen können kleinere Hüllkurven gefahren und eine auf zwei Zentimeter genaue Positionierung erreicht werden. Laserscanner kontrollieren die Umgebung und stellen zusammen mit Schaltleisten und Not-/AusTasten den Schutz von Personen, Ware und Fahrzeug sicher.

Im Projekt „Sichere autonome Logistik- und Transportfahrzeuge (SaLsA)“ erfolgten technologische Weiterentwicklungen, die den Begegnungsfall von FTS, Lkw und Personen im Außenbereich sicherer machen [8]. Im Außenbereich besteht das Sicherheitskonzept aus Radarsensoren, da dort keine Laserscanner zugelassen sind. Durch die kooperative Erfassung der Umgebung durch mobile und stationäre Sensorik wird die Sicherheit auch bei höheren Geschwindigkeiten (Wirtschaftlichkeit) realisiert. 


\subsubsection{Autonome Fahrzeuge für den Straßengütertransport außerhalb von Betriebsgeländen}

Die Entwicklung autonomer Fahrzeuge für den Gütertransport bzw. die Logistik außerhalb von Betriebsgeländen ist vor allem problemorientiert getrieben. Sogenannte Fahrroboter entstanden für den Einsatz in gefährlichen Situationen, z. B. zur Entschärfung von Munition, oder für den Einsatz in kaum zugänglichen Gebieten, wie beispielsweise für unterirdische Explorationen in der Tiefsee, für Arbeiten am Hang oder in dichtbewachsenen Wäldern oder auch in entlegenen Gebieten, beispielsweise für den Einsatz im Bergbau.

Sehr große automatisierte Lkw mit einer maximalen Beladung von 290 Tonnen kommen seit den 1990er-Jahren in einer der weltgrößten Eisenerzminen in Australien zum Einsatz [9]. Die Gründe für deren Entwicklung waren vor allem Schwierigkeiten bei dem zu akquirierenden Personal für den gefährlichen Schichtdienst im Outback und die hohen logistischen Anforderungen an die Personaleinsatzplanung und den Personentransfer.

Die Navigation erfolgt mit Radar und Lasern sowie durch den Einsatz von Wegpunkten zur Orientierung. Kontroll- und Eingreifmöglichkeiten sind über eine Betriebszentrale mittels WLAN möglich. Die Steuerung erfolgt durch GPS und Koppelnavigation wie bei einem Schiff oder Flugzeug durch laufende Ortsbestimmung (Ortung) indem der Kurs, Fahrt (Geschwindigkeit) und die Zeit ermittelt werden.

\subsubsection{Entwicklungen autonomen Fahrens und autonomer Fahrzeuge in den anderen Verkehrsträgern}

Auch die anderen Verkehrsträger sind durch eine Zunahme der Automatisierung gekennzeichnet, bei denen technische Systeme zur Stabilisierung, Navigation (z. B. digitale Karten) und Umweltwahrnehmung zunehmend zum Einsatz kommen.

Flugzeuge sind bereits seit Beginn des 20. Jahrhunderts mit Systemen zur Stabilisierung ausgerüstet, der Autopilot ist schon seit Langem die Regel und die ersten Drohnen fliegen in Deutschland für das Militär, die Polizei oder die Feuerwehr. Unmanned Aerial Systems oder auch Unmanned Aerial Vehicles (UAV) kommen in anderen Ländern auch im zivilen Bereich zum Einsatz, beispielsweise ersetzen sie Rancher und Inspektoren im Außenbereich. Drohnen überwachen Felder oder säen aus bzw. führen biologische Schädlingsbekämpfung durch. Kleine Drohnen kommen aber auch schon in Deutschland zum Einsatz, beispielsweise für die Begutachtung von Schäden durch Sturm oder Brand, in der Filmproduktion und bei Industrieinspektionen. Die Deutsche Bahn testete Drohnen für die Überwachung von Fahrzeugen und Infrastruktur. Weiter gibt es Pilotstudien im Bereich des Transports von Wirtschaftsgütern, insbesondere von Paketen. Derzeitige Drohnen könnten bei einer Tragfähigkeit von bis zu 2,5 Kilogramm und einer Reichweite von rund 15 Kilometern beispielsweise Fast-Food oder Medikamente ausliefern. Auch eine Drohne, durch die ein Defibrillator transportiert werden kann, wurde bereits getestet. Die Nutzung von Drohen für gewerbliche Zwecke über fünf Kilogramm ist mit einer Pauschal- 
genehmigung in vielen Bundesländern möglich; allerdings nicht in kontrollierten Lufträumen [10].

Die ersten Forschungsprojekte zur unbenannten Seeschifffahrt laufen derzeit (z. B. das europäische Projekt MUNIN). Unbemannte U-Boote sind schon seit Längerem im Einsatz. Konzepte für Drohnenschiffe werden von unterschiedlichsten Akteuren entwickelt. Ein Konzept ähnelt dem Platooning (s. Abschn. 18.3.4) im Straßenverkehr [11]. Im Unterschied zum Flugverkehr ist es möglich, dass bei stark befahrenen Abschnitten, beispielsweise beim Hafenanlauf, eine Besatzung jederzeit zusteigen kann. In der Regel werden diese Systeme - und werden es wohl auch zukünftig - nicht vollständig autonom agieren. Zwar können Ausweichmanöver selbstständig erfolgen, jedoch ist davon auszugehen, dass es eine zentrale Überwachung und Fern-Steuerung (Tele-Operation) geben wird, wobei beide Instanzen nicht identisch sein müssen.

Die Fernsteuerung von führerlosen Eisenbahnen findet bereits seit Jahren statt. Autonomes Fahren würde die freie Navigation mit einschließen und wäre nur für sehr kleine Einheiten zweckmäßig. Bisherige Ansätze für kleinere Gütertransporteinheiten auf der Bahn wie beispielsweise der CargoSprinter waren bisher nicht erfolgreich. Teilweise auch, weil eine schnelle Technik zur Zugbildung und -splittung nicht existiert und daher eine eigene Antriebstechnologie je Waggon notwendig ist. Dieses Manko griff das Konzept RailCab auf [12]. Hier nutzen autonome, mit Linearmotoren angetriebene Fahrzeuge das Rad-Schiene-System. Die Zugbildung erfolgt über eine elektronische Deichsel. Dies ist teuer und es gibt kaum sinnvolle Einsatzfälle, bei denen nicht der Lkw ebenso den Transport übernehmen könnte. Auf den Hauptläufen sprechen Effizienzgewinne durch den Massendegressionseffekt gegen ein nicht-vordisponiertes Routing und eine variable Konvoibildung. Es könnten sich aber ökonomisch sinnvolle Einsatzfälle gerade auf den nichtelektrifizierten Nebenstrecken ergeben. Bisher sind allerdings keine Studien bekannt, die den ökologisch-ökonomischen Vergleich unter Marktbedingungen anstellen.

\subsubsection{Zwischenfazit}

FTS bestehen aus einer Leitsteuerung, insbesondere zur Auftragsvergabe und Routenplanung, einem Kommunikationssystem und den Fahrzeugen [4]. Schon seit Langem existieren fahrerlose Transportsysteme und -fahrzeuge (FTS/FTF) für den innerbetrieblichen Transport mit geringen Automatisierungsstufen. Dabei hat der Automatisierungsgrad mit den technologischen Möglichkeiten zugenommen, allerdings anders als im öffentlichen Straßenverkehr. Beispielsweise sind die Sicherheitskonzepte, aufgrund der geltenden Maschinenrichtlinie anders ausgestaltet. In der Vergangenheit war es ausreichend, dass sich die FTS/FTF in der innerbetrieblichen Logistik vorwärts bewegen konnten. Dies erfolgt in der Regel in Szenerien, in denen die Menschen auf die fahrerlosen Fahrzeuge eingestellt waren. Teilweise gibt es eine räumliche Trennung von Mensch und Fahrzeug. Zusammenfassend kann festgehalten werden, dass sich die Sicherheitskonzepte auf ein Minimum beschränken, da die FTS/FTF bei geringen Geschwindigkeiten operieren und in bekannter Szenerie. 
Die technologische Weiterentwicklung bezog sich schwerpunktmäßig auf die korrekte Ausführung der Transportaufgabe und gegebenenfalls auf damit verbundene Aufgaben wie beispielsweise die Lastaufnahme bzw. -abgabe. Allerdings ist davon auszugehen, dass die Anforderungen an die Flexibilität der FTS/FTF-Systeme umso größer werden, je mehr sich die Produktion von der linear getakteten Fertigungsstraße entfernt und je flexibler die Produktions- und Kommissioniersysteme werden. Derzeit scheinen autonome Systeme basierend auf der Agententechnologie den Anspruch an die Verarbeitung eines zunehmenden Datenvolumens und einer steigenden Komplexität durch die Dezentralisierung von Datenbereitstellung und Entscheidungsfindung am ehesten leisten zu können.

Outdoor-Lösungen für den Gütertransport beruhen auf konventionellen Straßenfahrzeugen und sind bisher nur auf Betriebsgeländen im Regeleinsatz mit wenigen Einsatzfälle. Es ist aber eine ähnliche Entwicklung denkbar wie im Indoor-Bereich.

Es stellt sich also bei den FTS/FTF vor allem die Frage danach, wie sich die Autonomie der Einheiten weiterentwickelt. Automatische Steuerung und fahrerlose Fortbewegung sind per Definition schon immer Elemente von FTS/FTF gewesen. Der Grad an Autonomie ist definiert als die Anzahl der Freiheitsgrade. Diese hängen beispielsweise von der freien Wahl der Strecke und Geschwindigkeit ab, um sich selbstständig auch bei sich verändernden Umweltsituationen zu einem Ziel hin zu bewegen.

Der Einsatz von mobilen Maschinen bildet einen Schnittstellenbereich zwischen Arbeitsrobotern und Fahrzeugen. Ebenso wie bei den innerbetrieblichen Transportsystemen wird häufig die Transportaufgabe mit weiteren ,,produktiven“A Aufgaben kombiniert. Es handelt sich zum größten Teil um tele-operierte Systeme. Eine autonome Entscheidungsfindung für die Wegfindung ist also in der Regel nicht gegeben. Dies scheint auch in den meisten Einsatzfällen keinen Mehrwert zu schaffen.

Die konzeptionelle Ausgestaltung der Automatisierungslösungen hing in den Anfangsphasen sehr stark vom Anwendungskontext ab. Diese historischen Systemelemente finden sich auch in den neueren Konzeptansätzen in der Regel wieder, insbesondere hinsichtlich der Sicherheitskonzepte bzw. des Personenschutzes. Erst die mit den neuen Informationsund Kommunikationstechnologien möglichen dezentralen Konzepte lassen nun die unterschiedlichen Systemlösungen zusammenwachsen. Insbesondere dort, wo eine hohe Flexibilität und Geschwindigkeit gefordert wird, gleichen sich die technologischen Lösungen immer weiter an. Allerdings unterscheiden sie sich hinsichtlich der realisierten Freiheitsgrade der Autonomie.

\subsection{Anwendungsfälle im Bereich des autonomen Gütertransports}

Aufbauend auf den vorliegenden Erfahrungen werden im Folgenden potenzielle Einsatzfelder für autonome Fahrzeuge im Bereich des Gütertransports anhand der in Kap. 2 generisch beschriebenen Anwendungsfälle skizziert. Im Mittelpunkt steht der Transport eines aufgegebenen Förderguts im Straßengüterverkehr. Potenzielle Einsatzfelder weiterer Segmente des Wirtschaftsverkehrs wie beispielsweise der Personenwirtschaftsverkehr oder der 
Gütertransport, der im Rahmen der Produktionsfunktion anfällt (selbstfahrende Arbeitsmaschinen), werden nicht betrachtet.

Aufbauend auf einer konzeptionellen Beschreibung des jeweiligen Anwendungsfalls anhand der Merkmalsausprägungen erfolgt eine erste Einschätzung von Nutzen bzw. Chancen und Risiken einer derartigen Einführung. Vorab ist ein Exkurs zu den Automatisierungsgraden des Fahrens für den Gütertransport eingefügt, um die Ausdifferenzierung der Anwendungsfälle besser nachvollziehbar zu machen.

\subsubsection{Exkurs: Automatisierungsgrade des autonomen Gütertransports}

Für die Einordnung von Notwendigkeit und Nutzen eines konkreten Automatisierungsgrads im Gütertransport soll hier noch einmal kurz die Definition der Fahraufgabe nach [13] genutzt werden, wonach sich die Fahraufgabe folgendermaßen untergliedern lässt: Die sichere Ausführung der Fahraufgabe benötigt Informationen und Wissen über die Verkehrssituation auf der Bahnführungsebene, über die Fahrbahnoberfläche auf der Stabilisierungsebene sowie über das Straßennetz auf der Navigationsebene. Das sichere Führen des Fahrzeugs erfordert Lenken, Gas geben und Bremsen sowie Entscheidungen über die Geschwindigkeit und die Spur zur Längs- und Querführung des Fahrzeugs. Diese Entscheidungen werden in Abhängigkeit von Informationen über die Umweltbedingungen und dem damit verbundenen Handlungswissen getroffen.

Die Bundesanstalt für Straßenwesen kategorisiert automatisiertes Fahren in vier Entwicklungsstufen [14]: assistiertes Fahren (1), teilautomatisiertes Fahren (2), hochautomatisiertes Fahren (3) und vollautomatisiertes Fahren (4). Bei den drei erstgenannten Stufen übernimmt das System für einen gewissen Zeitraum und/oder in spezifischen Situationen Teilaufgaben der Fahraufgabe. Der Fahrer ist aber mindestens als Kontrollorgan vorhanden. Für die letzte Stufe des vollautomatisierten Fahrens (4) gehen [14] davon aus, dass das Fahrzeug frei navigiert und kein Fahrer (als Rückfallebene) benötigt wird.

Im Bereich des Gütertransports existieren fahrerlose Transportsysteme auch schon auf geringen Automatisierungsstufen. Mit einer Zwischenstufe zwischen (3) und (4) ist eine weitere relevante Ausprägung in der Transportpraxis zu beobachten: Das Fahrzeug ist hochautomatisiert und fahrerlos, eine freie Navigation erfolgt nicht. Häufig findet ein tele-operiertes Fahren statt, bei dem ein Operator das Fahrzeug von einer Zentrale aus fernsteuert.

\subsubsection{Anwendungsfälle des autonomen Gütertransports}

Assistierte und teilautomatisierte Systeme sind heute schon zum großen Teil in Serienfahrzeugen im Einsatz. Sie übernehmen über einen gewissen Zeitraum und/oder in spezifischen Situationen die Längs- und/oder Querführung des Fahrzeuges. Bei den assistierten Systemen erfolgt eine Warnung des Fahrers, bei den teilautomatisierten Systemen übernimmt 
das System die Regelung [3]. Durch eine Kontrollinstanz, im Straßenverkehr der Fahrer, wird das Fahrzeug permanent überwacht. Die bekanntesten Fahrerassistenzsysteme (FAS) sind das Antiblockiersystem (ABS) und das Elektronische Stabilitätsprogramm (ESP). Nach einem schweren Unglück sind seit November 2013 Spurwechsel- und -halteassistenten sowie Abstandsregeltempomaten frühzeitig für Neufahrzeuge verpflichtend geworden. Weitere Systeme sind in der Entwicklung oder bereits serienreif, wie Kippschutzsysteme, Abbiege- oder Einparksysteme. Sie zielen vor allem darauf ab, den Fahrer auf der Bahnführungsebene und der Stabilisierungsebene zu entlasten.

Hoch- und vollautomatisierte Fahrzeuge können darüber hinaus frei navigieren, sie sind situations- und infrastrukturunabhängig. Vollautomatisierte Systeme kommen ohne Fahrer aus. Es werden nicht nur Aktivitäten auf der Navigationsebene vom Fahrroboter mit übernommen, sondern auch diejenigen Aktivitäten, die notwendig sind, um das System wieder in einen risikominimalen Zustand zu versetzen, wenn Komponenten ausfallen.

Aus der Perspektive von Güterverkehr und Logistik stehen hier daher weniger die technologischen Lösungen für die Stabilisierungs- und Bahnführungsebene im Mittelpunkt. Vielmehr interessieren mögliche Anwendungsfälle mit und ohne Fahrer bzw. freier Navigation. Daher werden folgende Ausdifferenzierungen in Anwendungsfällen näher betrachtet:

1. Autobahnpilot als hochautomatisiertes Fahren mit Fahrer und freier Navigation,

2. Vehicle-on-Demand als vollautomatisierte Fahrt ohne Fahrer und mit freier Navigation,

3. Vollautomat mit Verfügbarkeitsfahrer - Follow-me-Fahrzeug als hochautomatisiertes Fahren ohne Fahrer und ohne freie Navigation (fehlender Anwendungsfall 3/4),

4. Valet-Parken als hochautomatisiertes Fahren ohne Fahrer und ohne freie Navigation (fehlender Anwendungsfall 3/4).

Im Folgenden werden die Anwendungsfälle vorgestellt und erste Argumente für und gegen den gewählten Autonomiegrad in Logistik und Güterverkehr aufgeführt. Es wird jeweils geprüft, inwieweit die Anwendungsfälle im Güterverkehrssystem mit den „Use Cases des Autonomen Fahrens“ (s. Kap. 2) im Individualverkehr deckungsgleich formuliert werden und wodurch sich Unterschiede und daraus andere Umsetzungsvoraussetzungen ergeben.

\subsubsection{Autobahnpilot mit Fahrer und freier Navigation}

Der Autobahnpilot bezeichnet den Anwendungsfall, in dem ein Fahrroboter zum Einsatz kommt, der Fahrer aber jederzeit verfügbar ist. Der Fahrer übergibt die Stabilisierungs- und die Bahnführungsebene idealerweise unter Angabe einer Zieladresse (und damit auch die Navigation) an den Fahrroboter. Der Einsatz eines Autobahnpiloten ist zwischen Autobahnauffahrt und -ausfahrt angedacht. Der Fahrer übernimmt bei unklaren Fahrsituationen (z.B. Baustellen). 
Der Nutzen des Autobahnpiloten wird vor allem in der Reduzierung von Unfällen und einem besseren Verkehrsfluss gesehen. Insbesondere bei Stau oder zähfließendem Kolonnenverkehr, aber auch bei langen, eintönigen Fahrten und durch den weiter zunehmendem Termindruck sind die Fahrer häufig an der Grenze ihrer Leistungsfähigkeit. Neben der Entlastung des Fahrers von stressbehafteten Fahrsituationen wird Arbeitszeit frei, die für andere Tätigkeiten genutzt werden kann. Hier gibt es Überlegungen, auch dispositive Tätigkeiten wie die Tourenplanung oder das Fuhrparkmanagement zu dezentralisieren.

Ein derartiger Einsatzfall ist bereits erprobt. Scania stellte im Jahr 2013 einen Lkw vor, der bis zu einer Geschwindigkeit von $50 \mathrm{~km} / \mathrm{h}$,,selbstständig beschleunigen, bremsen und lenken“ konnte [15]. Im Jahr 2014 ließ Daimler einen Lkw bis zu $85 \mathrm{~km} / \mathrm{h}$ autonom auf einem gesperrten Autobahnabschnitt zwischen anderen Fahrzeugen fahren [16].

Der konzipierte Autobahnpilot entspricht einer vollautomatisierten Fahrt. Aufgrund von Sicherheitsbedenken ist allerdings ein Verfügbarkeitsfahrer vorgesehen, sodass nur von einer hochautomatisierten Fahrt gesprochen werden kann. Die Einsatzhöchstgeschwindigkeit und die höhere maximal zulässige Gesamtmasse erfordern in ihrer Kombination andere Sicherheitskonzepte als im Individualverkehr.

Veränderungen werden vor allem beim Berufsbild des Fahrers erwartet. Bisher lernt er viel über die Fahrzeugtechnik und Ladungssicherung. Übernimmt der Fahrer weiterhin die technische Überprüfung des Fahrzeugs? Welche ökonomischen und ökologischen Einsparungen wären mit dem neuen Konzept verbunden?

\subsubsection{Vehicle-on-Demand als Autobahnfahrt ohne Fahrer mit freier Navigation}

Vehicle-on-Demand entspricht am ehesten dem Anwendungsfall, der im Bereich des Gütertransports als autonom, dezentral gesteuertes FTS/FTF bekannt ist. Ein Fahrersitz ist nicht vorgesehen. Allerdings kann sich in dem in Kap. 2 skizzierten Anwendungsfall das Fahrzeug bis zu einer Geschwindigkeit von bis zu $120 \mathrm{~km} / \mathrm{h}$ und auch in unbekannten Szenerien bewegen.

Es spricht einiges dafür, dass der Anwendungsfall ,,Vehicle-on-Demand“ den Wunschvorstellungen der Unternehmen nach autonomen Fahrzeugen im Güterverkehr sehr nahekommt: Der gut die Landessprache sprechende Fahrer, der bereit ist, die weiten Autobahnfahrten mit langen Abwesenheitszeiten bzw. unregelmäßigen Einsatzzeiten für einen geringen Lohn zu übernehmen, ist immer seltener zu finden. Übermüdete Lkw-Fahrer sind der häufigste Grund für schwere Unfälle. Das Andocken und das Manövrieren in engen Belieferungssituationen ist grundsätzlich für den Fahrer keine einfache Aufgabe.

Der Einsatz von Automatisierungstechnik könnte also von großem Nutzen sein. Allerdings wird sich die Freigabe aller Szenerien für schwere Lkw, insbesondere aufgrund von Sicherheitsbedenken und notwendigen Änderungen in der Supply Chain (s. Abschn. 18.4), noch einige Zeit hinauszögern. 
Mittelfristig eher realisierbar scheint der Autobahnpilot ohne Fahrer und mit freier Navigation zwischen Rendezvous-Punkten, beispielsweise zwischen Autohöfen auf Autobahnen, oder zwischen gut angebundenen Gewerbegebieten. Die Realisierungswahrscheinlichkeit dieses Anwendungsfalls könnte durch folgende Erweiterungskonzepte erhöht werden:

Sicherheitsbedenken gegenüber einem fehlenden Fahrer auf der Rückfallebene könnten durch separate $\mathrm{Zu}$ - und Abfahrten für autonome Fahrzeuge verringert werden, da Begegnungsfälle mit anderen Fahrzeugen auf ein Minimum reduziert werden würden. Durch die Nutzung einer eigenen Fahrspur (dedicated lane) durch autonome, gekoppelte Fahrzeuge könnten diese vermieden werden. Gleichzeitig könnte diese separate Fahrspur Ausgangspunkt für ein erweitertes Konzept mit alternativer Antriebstechnologie bilden, wenn diese Spur beispielsweise mit einer Oberleitung zur elektrischen Versorgung ausgestattet ist.

Die Kopplung von Fahrzeugen (Platooning) würde die Ausgangskonzeption, in der ein Verfügbarkeitsfahrer im vorausfahrenden Fahrzeug als Rückfallebene zur Verfügung steht, mit dem Erweiterungskonzept, in dem Fahrzeuge autonom ohne Fahrer fahren, kombinieren und die Vorteile beider Konzepte nutzen. Über ein Softwaresystem sind die Kolonnenfahrzeuge zusammengeschaltet. Für die sogenannte elektronische Deichsel bei hohen Geschwindigkeiten sprechen neben der besseren Ausnutzung der Straßeninfrastruktur vor allem die durch die Verringerung des Luftwiderstands erzielbaren Kraftstoffeinsparungen und Emissionsreduzierungen.

Mit der sogenannten elektronischen Deichsel wurden bereits seit Mitte der 1990erJahre mehrere Tests durchgeführt (siehe z. B. die europäischen Projekte „CHAUFFEUR I und II“, ,Safe Road Trains for the Environment (SARTRE)““, „Cooperative mobility solution for supervised platooning (COMPANION)“, das kalifornische „PATH-Programm“, das deutsche „KONVOI-Projekt: Entwicklung und Untersuchung des Einsatzes von elektrisch gekoppelten Lkw-Konvois auf Autobahnen“, das japanische „ITS Projekt“ der New Energy and Industrial Technology Development Organization (NEDO)). In diesen Projekten fuhren mehrere Lkw bzw. ein Konvoi von führendem Lkw und folgenden Pkw bis zu $90 \mathrm{~km} / \mathrm{h}$ sicher bei einem minimalen Abstand von vier Metern. Die Systeme basieren auf Radarsensoren, Stereokameras, dreidimensionalen Karten und meist auf den Datenaustausch mit anderen Fahrzeugen. Die bisherigen Versuche erfolgten immer mit einem Fahrer im Führungsfahrzeug und teilweise mit oder ohne Verfügbarkeitsfahrer in den Folgefahrzeugen. Es existieren auch bereits erste Ideen für ein fahrerloses Führungsfahrzeug.

In der Regel bauen diese Projekte auf bereits erprobter Technik auf: Die in Serien-Lkw verbauten Adaptive Cruise Control-Systeme dienen der Abstandskontrolle. Die Datenübertragung zwischen Führungs- und Folgefahrzeug erfolgt häufig mittels WLAN oder Infrarot.

Die ermittelten Kraftstoffeinsparungen bzw. $\mathrm{CO}_{2}$-Minderungspotenziale fielen in Abhängigkeit der gewählten Vehicle-to-Vehicle (V2V)-Kommunikation (bei „,versetztem Fahren"), der Art und des Aufbaus des Führungsfahrzeugs und der Folgefahrzeuge, des Abstands, der Geschwindigkeit sowie der Straßen- und Umweltbedingungen (Belag, Temperatur, Steigungen, Höhenlage) unterschiedlich aus. Sie betrugen um die fünf Prozent beim Führungs-Lkw und zehn bis 15 Prozent bei den Folge-Lkw [17]. 


\subsubsection{Vollautomat mit Verfügbarkeitsfahrer - Follow-Me-Fahrzeug}

In dem in Kap. 2 vorgestellten Anwendungsfall übernimmt das Fahrzeug als Vollautomat immer dann, wenn die Szenerie freigegeben ist. Der Fahrer ist immer dabei und kann gegebenenfalls die Fahrzeugführung übernehmen. Im Grunde ist es eine Ausweitung des Autobahnpiloten hinsichtlich der freigegebenen Szenerien und der zulässigen Geschwindigkeit und kommt dem autonomen Fahren sehr nahe.

Dieser Anwendungsfall ist aus wirtschaftlicher Perspektive nur dann interessant, wenn der begleitende Fahrer in dieser Zeit wertschöpfenden Tätigkeiten nachgehen kann. Gerade auf der sogenannten letzten Meile könnte die nächste Zustellung administrativ bereits während der Fahrt vor- bzw. nachbereitet werden. Derartige Fahrzeugkonzepte wären aber auch für den Einsatz im Personenwirtschaftsverkehr denkbar, beispielsweise für Tätigkeiten in der Altenpflege, in der Versicherungsvertretung etc., die ebenfalls mit Dokumentations- und Verwaltungstätigkeiten verbunden sind.

Als eine Vorstufe für einen Vollautomaten mit Verfügbarkeitsfahrer kann die bereits im Jahr 2011 vorgestellte Konzeptstudie „EmiL“ für ein teilautonomes Zustellfahrzeug dienen [18]. Bei diesem Fahrzeugkonzept muss der Zusteller nicht immer ein- und aussteigen, sondern kann über das Mobiltelefon das Fahrzeug anweisen, in Schrittgeschwindigkeit neben ihm herzufahren (Follow-me-Funktion). Bei allen unbekannten Situationen (z. B. Einmündungen, Kreuzungen) ist zusätzlich der DriveStick-Modus vorgesehen, mit dem bis zu sechs km/h schnell gefahren werden kann. Durch die Nutzung einer lokalen WLANVerbindung wird einem Signalabbruch, wie er bei GPS-Verbindungen befürchtet wird und auftreten kann, entgegengewirkt.

Im Rahmen des Forschungsprojektes konnte eine Einsparung an täglicher Zustellzeit von 40 Minuten ermittelt werden. Das typische Verletzungsrisiko durch Verdrehen und Umknicken beim Aussteigen wird verringert.

\subsubsection{Valet-Parken - Valet delivery}

Das Valet-Parken bezeichnet den Anwendungsfall, in dem der Fahrroboter das Fahrzeug an einen zuvor zugewiesenen freien Parkplatz autonom navigiert. Im vorgestellten Konzept in Kap. 2 wird davon ausgegangen, dass dies auch im öffentlichen Raum bis zu einer Geschwindigkeit von $30 \mathrm{~km} / \mathrm{h}$ möglich sein könnte. Ein Beispiel wäre die autonome Fahrt vom Wohnstandort des Fahrers zu einem definierten Parkplatz. Dieser Anwendungsfall ist für den Güterverkehr allerdings kaum vorstellbar, da es selten reservierte Lkw-Parkplätze mit einer Anbindung über das Nebenstraßennetz gibt.

Viel eher denkbar ist der Fall, dass in engen Innenstädten und in häufig nicht für große Lkw ausgelegten Anlieferzonen von Industrie und Handel der Fahrroboter das Einparken des Fahrzeugs oder das Andocken an die Rampe autonom übernimmt. Dadurch könnten teure Bagatellschäden verhindert werden. Der Fahrer wird von stressbehafteten Fahraufgaben entlastet, insbesondere dann, wenn er zugleich noch für die ermüdende, lange Auto- 
bahnfahrt zuständig ist. Valet delivery könnte aber auch dabei helfen, dass die Fahrer ihre Ruhezeiten einhalten können, wenn die „Last-Last-Mile“ ohne ihr Zutun abgewickelt werden könnte.

Ein anderer Anwendungsfall könnte die Baustellenbelieferung sein. Bei großen Bauvorhaben werden immer häufiger im Umfeld der Baustelle sogenannte Wartezonen für Lkw vorgehalten. Beispielsweise könnten die Abläufe optimiert werden, wenn sich die Fahrer bei größeren Betongussaktionen auf die Pendelfahrt konzentrieren könnten und die Wartezeiten entfallen. Der Fahrroboter übernimmt die Fahrt zwischen Wartestelle und Bauplatz.

Die Umsetzung hängt rechtlich sehr stark von der zwischen Wartestelle und Einsatzstelle gegebenen Entfernung und konkreten Szenerie ab. Ansonsten scheint die Umsetzung eher eine mentale als eine technische Herausforderung zu sein. Insbesondere aus der Perspektive des Fahrers, der dann in Szenerien zum Einsatz kommt, in dem ihm per se unterstellt wird, dass er sein Arbeitsgerät nicht beherrscht.

\subsection{Veränderungen in der Supply Chain durch einen höheren Automatisierungsgrad im Gütertransport}

Der systematische Nachvollzug der Veränderungen in der Supply Chain durch einen höheren Automatisierungsgrad im Gütertransport erfolgt entlang der in Abb. 18.1 dargestellten generischen Supply Chain. Jede Supply Chain bzw. Lieferkette besteht aus einer Aneinanderreihung der Aktivitäten „Rohstoffgewinnung“, „Verarbeitung/Produktion“, „Handel“" und den dazwischen stattfindenden logistischen Prozessen „Warenausgang (Umschlag)“, „Transport“ und „Wareneingang (Umschlag)““. Darüber hinaus ist unter heutigen Bedingungen die Produktions- und die Filiallogistik ebenso interessant, wenn es um Veränderungsprozesse aufgrund des nicht mehr vorhandenen Fahrers geht, da dort sehr viele sogenannte Added-Value-Services von den Logistik-Dienstleistern erbracht werden.

Der „Transport“ erfordert neben dem Fahren selbst von dem Fahrer die Kontrolle des Fahrzeugs, die Routenplanung sowie die Dokumentation und weitere administrative Tätigkeiten (Warenbegleitpapiere). Diese sind im grenzüberschreitendenden Verkehr noch umfangreicher (Zollabwicklung). Beim hochautomatisierten, fahrerlosen bzw. beim vollautomatisierten Fahren würde das häufige Problem der Lenk- und Ruhezeitüberschreitungen obsolet und Touren könnten anders geplant werden. Wenn der Transport hochautomatisiert erfolgt, wird erwartet, dass der Fahrer andere Aufgaben in dieser Zeit übernimmt. Er kann sich dann der Routenplanung, dem Fuhrparkmanagement oder der eigenen Erholung widmen [20].

Es kann also gleichzeitig zu einer Dezentralisierung und Parallelisierung von Funktionen kommen. Die Nutzung der Transportzeit für weitere Tätigkeiten ist allerdings nicht neu. Beispielsweise wurde die Behandlung von Postsendungen früher in Zügen vorgenommen (Postzug), Gleiches kann im Ausland beispielsweise auch bei den mobilen Depots (z. B. umgebaute Doppeldeckerbusse, „Floating Warehouse-Systeme“) beobachtet werden.

Grundsätzlich hat der Fahrer die Fahrzeugbe- und -entladung zu überwachen und teilweise sogar selbst durchzuführen. Im Warenausgang ist er für die Überprüfung der Fracht- 


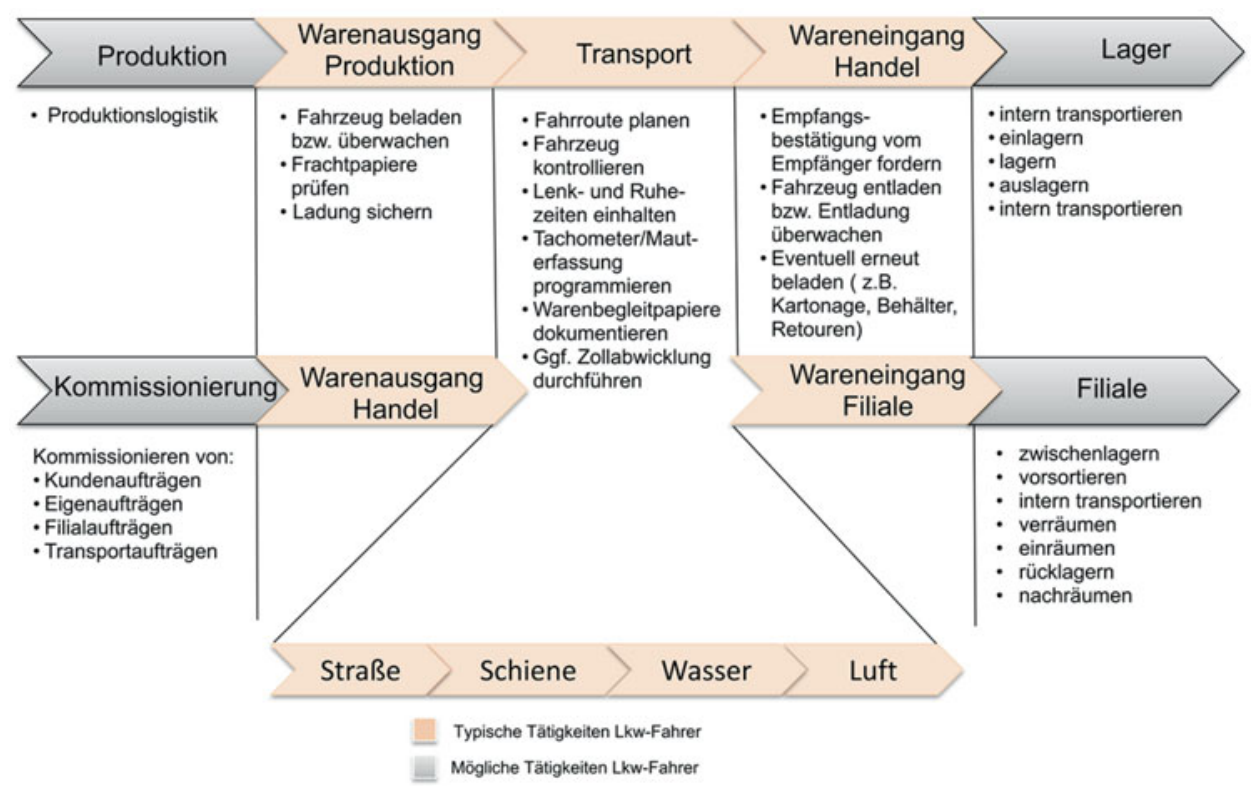

Abb. 18.1 Exemplarische fahrerrelevante Tätigkeiten einer generischen Supply Chain (Quelle: eigene Darstellung, basierend auf [19])

papiere und die Ladungssicherung zuständig. Im Wareneingang hat er eine Empfangsbestätigung zu fordern.

Im Bereich des Warenein- und -ausgangs sind heute Fahrer in vielen Lieferketten für die Entladung des Fahrzeugs zuständig. Ihre Aufgabe endet häufig an der Laderampe, teilweise aber erst nach dem Einräumen, Rücklagern oder Nachräumen am Band oder sogar nach Vorsortierung, internem Transport und der Verräumung der Waren in die Regale (teilweise in Zwischenlager oder Puffer). Wie früher ist es bei einigen Paket- und Briefzustellern Usus, dass der Fahrer die Kommissionierung der Aufträge selbst übernimmt.

Im Bereich der Produktion wären Einsatzfälle denkbar, beispielsweise im Werkverkehr, wo - ähnlich wie im innerbetrieblichen Bereich - Transportaufträge ausgeschrieben werden und sich autonome Lkw darum ,,bewerben“ und den Transportauftrag nach vordefinierten Kriterien gewinnen können. Hierfür wäre weder eine zentrale Leitsteuerung noch Wegfindung notwendig. Teilweise existieren bereits Güterschleusen, sodass im Falle des Gefahrenübergangs keine Personen anwesend sein müssen.

Würde der Fahrer das Fahrzeug nicht mehr ,begleiten“, müssten diese Tätigkeiten von anderen übernommen werden. Die Unternehmen müssten wieder eigene Beschäftigte für diese Tätigkeiten einstellen bzw. anlernen. Denkbar wäre aber auch, dass sich für die Logistik-Dienstleister hier noch ein weiteres Geschäftsmodell ergibt. Es kann also wieder zu einer Aufwertung von Arbeit allgemein und der Schaffung von lokaler Arbeit vor allem im urbanen Raum kommen. In anderen Fällen wäre es aber auch denkbar, dass der Automatisierungsgrad im Warenein- und -ausgang weiter zunimmt. 
Veränderungen in den Transportsystemen selbst sind vor allem an der Schnittstelle zwischen unbemannter und bemannter Fahrt zu erwarten sowie im Aufgabenprofil der Fahrer. Die sich ergebenden prozessualen Veränderungen im Bahn- und Schiffsverkehr sowie für das Tätigkeitsspektrum der Menschen scheinen nicht so gravierend zu sein. Der Fahrer entfällt und wird durch einen Fahrroboter ersetzt. Die restliche Technik von Bahn und Schiff stehen bisher unter einem Veränderungsvorbehalt. Allerdings könnten neue Berufsbilder entstehen, wenn beispielsweise im Seeverkehr der Sicherheitsaspekt gegen Angreifer höher gewichtet wird als die Navigation.

\subsection{Erste einzelwirtschaftliche Einschätzungen von automatisierten Systemen in der Gütertransportkette}

Fehlendes Fahrpersonal, energiesparende Fahrweise, hohe Zuverlässigkeit und effiziente Unfallvermeidung sind aus einzelwirtschaftlicher Perspektive gewichtige Gründe, autonome Systeme in der Gütertransportkette einzusetzen. In einigen Industrien ist der Materialfluss inzwischen vom Wareneingang über die Produktionsstraßen bis hin zum Warenausgang hochautomatisiert und fahrerlos gestaltet und weist damit keine Unterbrechungen und keine Liegezeiten auf. Auch die Beschickung und Entnahme aus den Lägern erfolgt in einigen Branchen hochautomatisiert und fahrerlos. Teilweise sind die Steuerungen von Lager und Produktion aufeinander abgestimmt und reichen vom automatisierten Wareneingang zum automatisierten Warenausgang.

Mit der Entwicklung der Radio Frequency Identification (RFID)-Technologie und der Wiederentdeckung der alten Idee von Leonardo da Vinci, von der Natur zu lernen (Bionik), wurde die Debatte um Selbststeuerung inspiriert und führte zu einer intensiven Diskussion um Dezentralisierung und Autonomie der Entscheidungsfindung technischer Systeme. Unterstützt durch die technologischen Entwicklungen von kostengünstigen Sensoren und internetgestützten Softwaresystemen hat der Automatisierungsgrad daher immer weiter zugenommen. Die neuesten Systeme in Produktion und Lager setzen auf die Selbststeuerung (Internet der Dinge) der Fahrzeuge und Fördersysteme, die autonom auf veränderte Arbeitsanforderungen, Zustände und Umfeldbedingungen reagieren können.

Ausgehend von der innerbetrieblich in bestimmten Branchen stark vorangeschrittenen Automatisierung von FTS/FTF ist mit einer weiteren Verschiebung der menschlichen Aktivitäten hin zu konzeptionellen und überwachenden Tätigkeiten zu rechnen. Durch den Einsatz von fahrerlosen Fahrzeugen im gesamten logistischen System ist damit zu rechnen, dass in vielen Supply Chains Prozesse reorganisiert werden müssen (s. Abschn. 18.4). Allerdings wird die Automatisierung wie bisher verstärkt in einzelnen Branchen zunehmen, da Aufwand und Nutzen nicht überall in einem angemessenen Verhältnis stehen werden und sich gerecht verteilen lassen.

Innerhalb des Transportsystems im öffentlichen Raum würden das Entfallen des Fahrers und/oder dessen veränderte Rolle zu zusätzlichen Schnittstellen führen, die gestaltet werden müssen. Das Zu- und Aussteigen des Fahrers bedingt zusätzliche Halte. Im innerbetrieb- 
lichen Bereich gibt es beispielsweise mit den Kommissionierern bereits umfangreiche Erfahrungen. In den Verkehrssystemen liegen diese Erfahrungen nur bedingt vor. Im Seeverkehr ist es beispielsweise durchaus üblich, dass beim Hafeneinlauf ein Hafenkapitän das Steuer übernimmt. Im Straßengüterverkehrssystem ist bisher der Begegnungsverkehr erprobt, wo in aller Regel der Auflieger bzw. Hänger gewechselt wird und nicht der Fahrer. Diese Systeme haben viele Vorteile wie schnelle Transferzeiten der Waren und Güter und damit die Ausweitung der produktiven Zeiten der Fahrzeuge. Zudem verbessern sich die Arbeitsbedingungen für die Fahrer, die täglich zu ihrer Niederlassung zurückkehren. In der Praxis funktioniert dies jedoch nur in großen Netzen bzw. auf paarigen Relationen und mit vertrauenswürdigen und zuverlässigen Partnern. Dies gilt nicht nur für die Pünktlichkeit, sondern insbesondere auch hinsichtlich der Ladungssicherung. Die größte Verbreitung haben diese Systeme bisher bei den großen Integratoren bzw. KEP-Dienstleistern mit standardisierten Sendungen gefunden.

Für die Umsetzung von fahrerlosen Transportketten im Straßengüterverkehr sind also neben der Frage von technischer Kompatibilität der autonomen Fahrzeuge mit der Infrastruktur und mit anderen Fahrzeugen oder auch mit Platooning-Lkw immer auch Kostenund Haftungsfragen im Transportrecht zu klären.

Kommt es zu Schäden an der Ware oder am Fahrzeug aufgrund von Systemausfällen, könnte die Produkthaftung greifen (s. im Detail Kap. 25). Daraus folgt, dass die Fahrroboter bestehend aus Hardwarekomponenten (Sensoren, Prozessoren und Aktoren) und Softwareelementen so aufgebaut werden müssen, dass mögliche Schäden den jeweiligen Komponenten und damit deren Produzenten direkt zurechenbar sind.

\subsection{Erste gesamtwirtschaftliche Einschätzungen von automatisierten Systemen in der Gütertransportkette}

Auch aus gesamtwirtschaftlicher Perspektive könnten automatisierte Systeme einen Lösungsansatz für strukturelle Defizite im heutigen Verkehrswesen leisten. In Zeiten knapper öffentlicher Haushalte ist künftig nicht mit einem deutlichen Ausbau von Verkehrsinfrastruktur, insbesondere von Schiene und Binnenschiff, zu rechnen.

Mit dem automatisierten Fahren könnte die vorhandene Straßeninfrastrukturkapazität durch den geringeren Platzbedarf und durch die gleichmäßigere Geschwindigkeit mindestens verdoppelt werden. Darüber hinaus könnte ein Beitrag zum Klimaziel geleistet werden, da automatisiertes Fahren den Treibstoffverbrauch reduziert. Bereits heute leisten Fahrerassistenzsysteme (FAS) einen erheblichen Beitrag zur Reduzierung von Unfällen. Deren Anzahl könnte durch hoch- bzw. vollautomatisierte Fahrzeuge weiter verringert werden, da sie Stauenden erkennen können, riskante Überholmanöver vermeiden und auch keine Geisterfahrten unternehmen. Allerdings sind die rechtlichen Haftungsfragen, wenn es dann doch zu einem Unfall käme, noch nicht vollständig gelöst.

Im Falle des Platooning könnte im Führungsfahrzeug ein professioneller Fahrer zum Einsatz kommen, so wie es in den meisten Projekten auch angedacht ist. Dies hätte den 
Vorteil, dass sich beispielsweise auch ungeübte oder weniger belastbare Langstreckenfahrer (Fahranfänger, Ältere) in eine Kolonne einordnen könnten. Dies würde jedoch bedingen, dass Güter- und Personenverkehrsfahrzeuge miteinander kommunizieren könnten.

Allerdings liefert nicht nur der Technikdeterminismus gewichtige Gründe gegen automatisierte Straßenfahrzeuge: Effizienzerhöhungen im Straßenverkehr rufen Konflikte mit den Massentransportmitteln hervor. Automatisiertes Platooning steht in direkter Konkurrenz mit der Bahn, wie zuvor schon der Lang-Lkw. Auch wenn es derzeit in Deutschland einen Berufskraftfahrermangel gibt, so würde doch die Anzahl an Berufskraftfahrern insgesamt abnehmen, und in anderen Ländern würden aus gesamtwirtschaftlicher Perspektive dringend benötigte Arbeitsplätze entfallen.

Auch die Akzeptanz der Bevölkerung ist gegenüber vollautomatisierten Systemen beschränkt - nicht zuletzt aufgrund regelmäßiger Berichte von in Fahrzeugen ausgefallenen mechanischen oder elektronischen Bauteilen oder aus Angst vor abreißenden Datenverbindungen. Autonome Fahrzeuge müssen zudem mit einer entsprechenden, zuverlässigen Software (Künstlichen Intelligenz) ausgestattet sein, die auf alle Eventualitäten reagieren kann - insbesondere dort, wo Menschen und Tiere auf den Straßen unterwegs sind.

Aus rechtlicher Perspektive stellen Fahrroboter außerhalb der Werkshallen eine Neuheit da. Bisherige FTS/FTF wurden nach der Maschinenrichtlinie behandelt, mit entsprechenden Ausgestaltungen von Sicherheits- und Personenschutzkonzepten. Eine Ausweitung des Aktionsraums (privat/öffentlich) und der Einsatzgeschwindigkeit (Schrittgeschwindigkeit bis Autobahnfahrt) der Systeme könnte neue bzw. eine Anpassung der bestehenden rechtlichen Regelungen notwendig machen.

Für den Einsatz von autonomen Fahrzeugen im Straßenverkehr wird gefordert, die rechtlichen Einschränkungen durch die „Wiener Straßenverkehrskonvention“ abzuschaffen, die vorsieht, dass ein Fahrer jederzeit die Kontrolle über sein Fahrzeug behalten müsse. Hier gibt es inzwischen eine neuere Entwicklung in den Vereinigten Staaten, deren Rechtsprechung insofern angepasst wurde, als sichergestellt ist, dass das System ,can be overridden or switched off by the driver". Sollte sich diese Rechtsauffassung auch in Europa durchsetzen, würde dies bedeuten, dass es immer eine übergeordnete Instanz geben muss, die in das Fahrzeug steuernd eingreifen kann. Im Bereich des Bahn-, See- und Luftverkehrs werden die Systeme heute schon so ausgelegt und für den Bahn- und Luftverkehr existieren akzeptierte übergeordnete Instanzen. Für den Straßengüterverkehr muss eine derartige Instanz erst geschaffen werden. Dafür muss die notwendige Akzeptanz hergestellt werden.

\subsection{Fazit und Ausblick}

Seit den 1960er-Jahren sind Fahrerlose Transportsysteme (FTS) in der innerbetrieblichen Logistik in Deutschland im Einsatz. Allerdings werden diese Entwicklungen weder medial noch in der Verkehrssystemforschung bisher ausreichend wahrgenommen. In der innerbetrieblichen Logistik kommen beispielsweise Transponder als Wegmarken zum Einsatz, die auch Informationen speichern können. Wenn sich Fahrzeuge im öffentlichen Raum 
immer alle Umgebungsmerkmale und -besonderheiten jeder Fahrstrecke merken müssten, würden entsprechend kostenintensive Hard- und Softwaresysteme notwendig. Diese könnten vermieden werden, wenn Wegmarken bestimmte Informationen bereithielten, die nur temporär ausgelesen werden müssten. Übertragen auf das Verkehrssystem stellt sich hier die Frage, wie die Straßeninfrastruktur verändert werden müsste.

Vollautomatisiertes Fahren eröffnet die Möglichkeit, Fahrzeuge ohne menschlichen Eingriff zwischen Quelle und Ziel zu steuern. Der Fahrroboter übernimmt nicht nur die Stabilisierung und Bahnführung, sondern auch die Navigation und trägt damit auch die Verantwortung für die unfallfreie Fahrt.

Die Bestandsaufnahme hinsichtlich der Entwicklung autonomer Straßenverkehrssysteme hat deutlich gemacht, dass die Betrachtung von Personen- und Gütermobilität wesentlich integrierter erfolgen muss. Ebenso spannend wäre eine vertiefende Betrachtung der autonomen Systementwicklung im Bereich der anderen Verkehrsträger. Der Autopilot in Flugzeugen ist schon seit Langem die Regel. Bereits zu Beginn des 20. Jahrhunderts kamen dort Systeme zur Stabilisierung zum Einsatz. Die ersten Drohnen fliegen. Die ersten Forschungsprojekte zur unbenannten Schifffahrt auf europäischer Ebene laufen. Auch die Fernsteuerung von Eisenbahnen findet bereits seit Jahren statt. Neu ist die Qualität der autonomen Entscheidungsfindung bei veränderter Umweltsituation oder neuem bzw. verändertem Fahr- bzw. Transportauftrag. Die Ausweitung der Einsatzgebiete, beispielsweise im Luftverkehr durch die Drohnen, zeigt die Notwendigkeit auf, dass sich Transportaufträge vollkommen neu über die Verkehrsträger verteilen können. Hier besteht Forschungsbedarf.

Viele spannende Einblicke und Neuerungen wären zu erwarten, wenn das große Feld des Personenwirtschaftsverkehrs näher untersucht werden würde. Ebenso wie beim Güterverkehr geht es um Mobilität in Ausübung des Berufs. Da die Raumüberwindung nur Mittel zum Zwecke der Leistungserbringung am angefahrenen Ort ist, wird die Fortbewegung selbst in den meisten Fällen sehr viel pragmatischer betrachtet als im Individualverkehr.

Die Zusammenführung der Teilszenarien von einzelnen, autonomen logistischen Prozessen zu einem Szenario einer ganzheitlich autonomen Supply Chain bzw. Lieferkette von der automatisierten Rohstoffgewinnung, über alle Produktions- und Logistikstufen hinweg bis hin zur Belieferung des Endkunden erfordert eine integrierte Betrachtung. Technologisch wurden hier in den letzten Jahren enorme Fortschritte erzielt. Die Forschungsarbeiten müssen daher vor allem hinsichtlich des (notwendigen) Grads der Automatisierung einzelner logistischer Prozesse sowie der Feststellung von einzel- und gesamtwirtschaftlichen Nutzen und Kosten intensiviert werden.

Aufgrund der offenen rechtlichen Fragen und der fehlenden Akzeptanz innerhalb der Bevölkerung und teilweise auch unter den Unternehmensentscheidern für den Einsatz autonomer Systeme im Straßengüterverkehr (und teilweise auch in den anderen Verkehrssystemen) scheint hier nur eine schrittweise Einführung sinnvoll und realistisch. Daher wird es auf die richtige kaskadische Ausgestaltung und Implementierungsstrategie ankommen. Allerdings fehlen bisher spezifische Betrachtungen der jeweiligen Umsetzungsaspekte der skizzierten Anwendungsfälle. 
Auf den ersten Blick erscheint es durchaus sinnvoll, einige der derzeit diskutierten Lösungsansätze zur Kapazitätserhöhung der Infrastruktur, Ressourcenschonung und des Umweltschutzes zu kombinieren. Der Beginn der Einführung des autonomen Fahrens in geschlossenen Systemen (beispielsweise Systemverkehren) und in überschaubaren Szenerien (z.B. Autobahn, Flughafenvorfeld, Hafen) wird Vertrauensvorbehalte und fehlende Standardisierungen überwinden helfen. Die schrittweise Umsetzung des Platooning, ausgehend von bemannten Führungs- und Folgefahrzeugen, könnte die notwendige Akzeptanz in der Bevölkerung schaffen. Zu prüfen ist, ob die gleichzeitige Einführung von Oberleitungs-Lkw und damit die Schaffung einer separaten Fahrspur unter Kapazitäts- und Sicherheitsaspekten sinnvoll wäre.

Allerdings wirft auch das Platooning mehrere Anschlussfragen auf, insbesondere hinsichtlich der Entscheidungsfindung und der Nutzenverteilung: Wenn die Kraftstoffeinsparung von der Position im Konvoi abhängig ist, wer bestimmt über den Platz in der Kette? Wenn der Konvoi ohne Fahrer in den Folgefahrzeugen fahren würde, wer bezahlt den Fahrer im Führungsfahrzeug? Braucht der Führungsfahrer eine spezielle(re) Ausbildung gegenüber den anderen Fahrern? Vor der Einführung muss aber auch geklärt werden, wie andere Platooning-Fahrzeuge identifiziert werden könnten. Dafür müsste ein gemeinsamer Standard entwickelt werden, der auch sicherstellt, dass die anderen Fahrzeuge vertrauensvoll sind. Hier greifen der weitere Forschungsbedarf und die Handlungsempfehlungen eng ineinander.

Die Veränderungen in der Supply Chain und im Güterverkehr zeigen ein sehr ambivalentes Bild, das genauer untersucht werden muss. Für das Verständnis, die Bewertung und die Einordnung ist zu klären, welche technologischen Veränderungen und Herausforderungen sich für die Fahraufgabe ergeben. Zudem gilt es, die Vor- und Nachteile des Einsatzes von automatisierten gegenüber konventionellen Fahrzeugen und deren Integrationsfähigkeit in die bestehende Arbeitsumgebung genauer zu analysieren, indem hinsichtlich verschiedener Tätigkeitsprofile von Unternehmen unterschieden wird. Bisher ist die Frage unbeantwortet, was alles autonom transportiert werden kann und welcher Autonomisierungsgrad überhaupt von der Wirtschaft hinsichtlich Notwendigkeit, Kosten und Flexibilität akzeptiert werden würde.

Gleichzeitig ergeben sich aber auch neue Möglichkeiten für innovative Geschäftsmodelle, deren Konzeptualisierung und Bewertung bisher allerdings ausstehen. Auch könnten sich Alternativen für Einsatzbereiche ergeben, deren Herausforderungen bis heute ungelöst erscheinen. Ein Beispiel ist die Ver- und Entsorgung von innerstädtischen Standorten (Stichwort „City-Logistik ${ }^{6}$ ), die sehr konfliktträchtig und kostenintensiv ist. Der Einsatz von autonomen Straßenverkehrsfahrzeugen, gekoppelt mit weiteren Komponenten (z.B. Güterschleusen), könnte beispielsweise eine zeitliche Entzerrung der Personen- und Güterverkehrsfahrten ermöglichen.

Parallel dazu sollten jedoch auch die Chancen und Risiken der zunehmenden Automatisierung des Transports aus einer gesamtwirtschaftlichen, länderübergreifenden Perspektive genauer analysiert werden. Dazu gehören nicht nur rechtliche Fragen und Standardisierungsnotwendigkeiten, sondern auch der weitere Wegfall von Arbeitsplätzen, die 
keinen höheren Bildungsabschluss voraussetzen. Hier muss eine Betrachtung von Arbeitsalternativen stattfinden. $\mathrm{Zu}$ wenig untersucht ist bisher auch, welche Konflikte beispielsweise zwischen dem autonomen Straßengütertransport (im Konvoi) und den Massentransportmitteln auftreten können. Zudem bedarf die Diskussion unbedingt der thematischen Erweiterung um eine ganzheitliche Umwelt- und Ressourcenbewertung.

\section{Literatur}

1. Vahrenkamp, R.: Von Taylor zu Toyota: Rationalisierungsdebatten im 20. Jahrhundert. 2. korrigierte und erw. Auflage. Josef Eul Verlag: Lohmar-Köln (2013)

2. Ullrich, G.: Fahrerlose Transportsysteme. 2. Auflage, Springer Fachmedien, Wiesbaden (2014)

3. Ullrich, G.: Effizientes Fulfillment mit Fahrerlosen Transportfahrzeugen. DS Automotion. http:// www.ds-automotion.com/uploads/tx_sbdownloader/hermes.pdf(2012). Zugegriffen: 04. Juli 2014

4. Schwarz, C., Schachmanow, J., Sauer, J., Overmeyer, L., Ullmann G.: Selbstgesteuerte Fahrerlose Transportsysteme - Self Guided Vehicle Systems. Logistics Journal (2013). doi: 10.2195/ 1j_NotRev_schwarz_de_201312_01

5. Sauer, J., Ullmann, G.: Schlussbericht dem geförderten Vorhaben 17237 N. Dezentrale, agentenbasierte Selbststeuerung von Fahrerlosen Transportsystemen (FTS). Bundesvereinigung Logistik e.V.. http://www.bvl.de/files/441/481/17237_Schlussbericht_FTS-Selbststeuerung_20131002.pdf (2013). Zugegriffen: 27. Juli 2014

6. Hamburger Hafen und Logistik AG: Wer hat's erfunden? Hamburger Hafen und Logistik AG. http://hhla.de/de/container/altenwerder-cta/das-projekt-agv.html (2014). Zugegriffen: 02. Juli 2014

7. Dermuth, R.: Fahrerloser LKW in einer Molkerei. GÖTTING. http://www.goetting.de/ news/2012/molkerei (2013). Zugegriffen: 02. Juli 2014

8. Kerner, S.: SaLsA - Sichere autonome Logistik- und Transportfahrzeuge. Fraunhofer IML. http://www.iml.fraunhofer.de/de/themengebiete/automation_eingebettete_systeme/Forschung/ salsa.html (2014). Zugegriffen: 27. Juni 2014

9. Komatsu Ltd.: Autonomous Haul System (AHS). Komatsu Ltd. http://www.komatsu.com.au/ AboutKomatsu/Technology/Pages/AHS.aspx(2005). Zugegriffen: 02. Juli 2014

10. Wirth, N.: Zivile Drohnen heben ab - auch an der Börse? Trendlink.com. http://www.trendlink. com/aktienanalysen/aktien/zivile_Drohnen/78-Zivile_Drohnen_heben_ab_-_auch_an_der_Boerse (2014). Zugegriffen: 22. Juli 2014

11. Hegmann, G.: Rolls Royce glaubt fest an Drohnenschiffe. Welt.de. http://www.welt.de/wirtschaft/article123352845/Rolls-Royce-glaubt-fest-an-Drohnenschiffe.html (2013). Zugegriffen: 30. Juli 2014

12. Böcker, J., Henke, C., Rustemeier, C., Schneider, T., Trächtler A.: Rail Cab - Ein Schienenverkehrssystem mit autonomen, Linearmotor getriebenen Einzelfahrzeugen. Universität Paderborn. http://wwwlea.uni-paderborn.de/fileadmin/Elektrotechnik/AG-LEA/forschung/ veroeffentlichungen/2007/07ETG_Henke_Rustemeier_Schneider_Boecker_Traechtler.pdf (2007). Zugegriffen: 30. Juli 2014

13. Donges, E. 1982. Aspekte der Aktiven Sicherheit bei der Führung von Personenkraftwagen. Automobil-Industrie, 2, S. 183-190

14. Gasser, T.M.: Rechtsfolgen zunehmender Fahrzeugautomatisierung. Berichte der Bundesanstalt für Straßenwesen 83. (2012)

15. Brünglinghaus, C.: Scania erforscht das automatisierte Lkw-Kolonnenfahren. Springer für Professionals. http://www.springerprofessional.de/scania-erforscht-das-automatisierte-lkw-kolonnenfahren/4909638.html (2014). Zugegriffen: 04. Juli 2014 
16. Grünweg, T.: Autonom fahrender Lkw: Laster ohne Lenker. Spiegel Online. http://www.spiegel. de/auto/aktuell/autonome-lkw-neue-technik-soll-fernfahrer-entlasten-und-sprit-sparen-a-978960. html (2014). Zugegriffen: 04. Juli 2014

17. Tsugawa, S.: Energy ITS: What We Learned and What We should Learn. Onlinepubs.trb.org. http:/onlinepubs.trb.org/onlinepubs/conferences/2012/Automation/presentations/Tsugawa.pdf (2012). Zugegriffen: 30. Juli 2014

18. Bundesministerium für Umwelt, Naturschutz und Reaktorsicherheit (Hrsg.): Abschlussbericht zum Verbundvorhaben Erprobung nutzfahrzeugspezifischer E-Mobilität - EmiL. erneuerbarmobil.de. http://www.erneuerbar-mobil.de/de/projekte/foerderprojekte-aus-dem-konjunkturpaket-ii-2009-2011/wirtschaftsverkehr-feldversuche/abschlusberichte-wirtschaftsverkehr/abschlussbericht-emil.pdf (2011). Zugegriffen: 02. Juli 2014

19. Bieber/Deiß 2000 Bieber, D.: Schnittstellenoptimierung in der Distributionslogistik: innovative Dienstleistungen in der Wertschöpfungskette. SSOAR. http://www.ssoar.info/ssoar/bitstream/ handle/document/9993/ssoar-2000-bieber_et_al-schnittstellenoptimierung_in_der_distributionslogistik.pdf (2000) Zugegriffen: 17. Juli 2014

20. Daimler AG: Weltpremiere: Daimler Trucks präsentiert mit autonom fahrenden „Mercedes-Benz Future Truck 2025“ den Lkw der Zukunft. daimler.com. http://www.daimler.com/Projects/c2c/ channel/documents/2495102_Daimler_IRRelease_03072014_de.pdf (2014). Zugegriffen: 04. Juli 2014 